\title{
Noise Characterization: Keeping Reduction Based Per- turbed Quantum Walk Search Optimal
}

\author{
Chen-Fu Chiang ${ }^{1, *}$ and Chang-Yu Hsieh ${ }^{2, * *}$ \\ ${ }^{1}$ Department of Computer Science, State University of New York Polytechnic Institute, Utica, \\ NY 13502, USA. \\ ${ }^{2}$ Department of Chemistry, Massachusetts Institute of Technology, Cambridge, MA 02139, USA. \\ Singapore-MIT Alliance for Research and Technology (SMART) Centre, Singapore 138602.
}

\begin{abstract}
In a recent work by Novo et al. (Sci. Rep. 5, 13304, 2015), the invariant subspace method was applied to the study of continuous-time quantum walk (CTQW). In this work, we adopt the aforementioned method to investigate the optimality of a perturbed quantum walk search of a marked element in a noisy environment on various graphs. We formulate the necessary condition of the noise distribution in the system such that the invariant subspace method remains effective and efficient. Based on the noise, we further formulate how to set the appropriate coupling factor to preserve the optimality of the quantum walker.
\end{abstract}

\section{Introduction}

Quantum walks (QW) can be formulated in discrete time (DTQW) [1] and continuous time [2] (CTQW) versions. The connection between discrete time quantum walk and continuous time quantum walk has been well studied. It was shown that continuous-time quantum walk can be obtained as an appropriate limit of discrete-time quantum walks [3]. In this work, we adopt the invariant subspace method from [4], which allows us to perform a dimensionality reduction to simplify the analyses of CTQW on a uniform complete multi-partite graph. The invariant approach reduces the original complex graph to a much smaller graph. The reduced graph captures the dynamics of the original graph such that we can investigate, such as the optimality of a quantum walk search, and test by using current quantum technology. Furthermore, the analysis becomes more transparent and the dynamics of the walker can be more intuitively understood on an abstract level.

The contribution from this work is as follows. We extend reducible graphs from complete graphs, complete bipartite graphs and star graphs discussed in [4] to uniform complete multi-partite graphs. We further parameterize the coupling factor based on the configuration of a given uniform complete multi-partite graph to keep the CTQW search on uniform complete multi-partite graphs optimal. Finally we characterize the error patterns such that the perturbed Hamiltonian remains reducible and our parameterized coupling factor keeps

\footnotetext{
*e-mail: chiangc@sunyit.edu

**e-mail: changyuh@mit.edu
} 
the quantum walker search remain optimal.

In section 2, we describe the work that applies the Lanczos algorithm to perform dimensionality reduction to obtain the right form of the reduced adjacency matrix. We breifly show the theorems to show (a) how to choose the correct coupling factor based on the given parameters (configuration) on a reduced adjacency matrix and (b) the optimality is preserved once transformed back to the original adjacency matrix. In section 3, under three types of errors, systematic disorder, static diagonal disorder, and reducible non-diagonal disorder, we characterize the errors such that systematic dimensionality reduction to a small Hamiltonian is feasible. We draw our conclusion in section 4 .

\section{Background}

Throughout the text we refer to a multi-partite graph as a $P$-partite with a slight twist on the standard notation. The difference is that the whole graph has actually $P+1$ partitions where the extra one partition is the partition that contains the solution (marked vertex). A uniform complete P-partite graph (UCPG) can be denoted as $G\left(V_{0}, V_{1}, \ldots, V_{P}\right)$. Let $V$ be the union of all partitions. A UCPG is a graph with $P+1$ partitions of vertices with the following properties: (1) each vertex $v_{i}$ in vertex partition $V_{j}$ connects to all other vertices in vertex partition $V_{k}$ as long as $j \neq k$ (2) except vertex partition $V_{0}$, each of the vertex partitions has the same size. Let the size of the vertex partition $V_{j}$ be $m_{j}$, i.e. $m_{j}=\left|V_{j}\right|$. Since if a UCPG G has $N$ vertices, then we know $P \times m_{1}+m_{0}=N$ since $m_{1}=m_{2}=\cdots=m_{P}$.

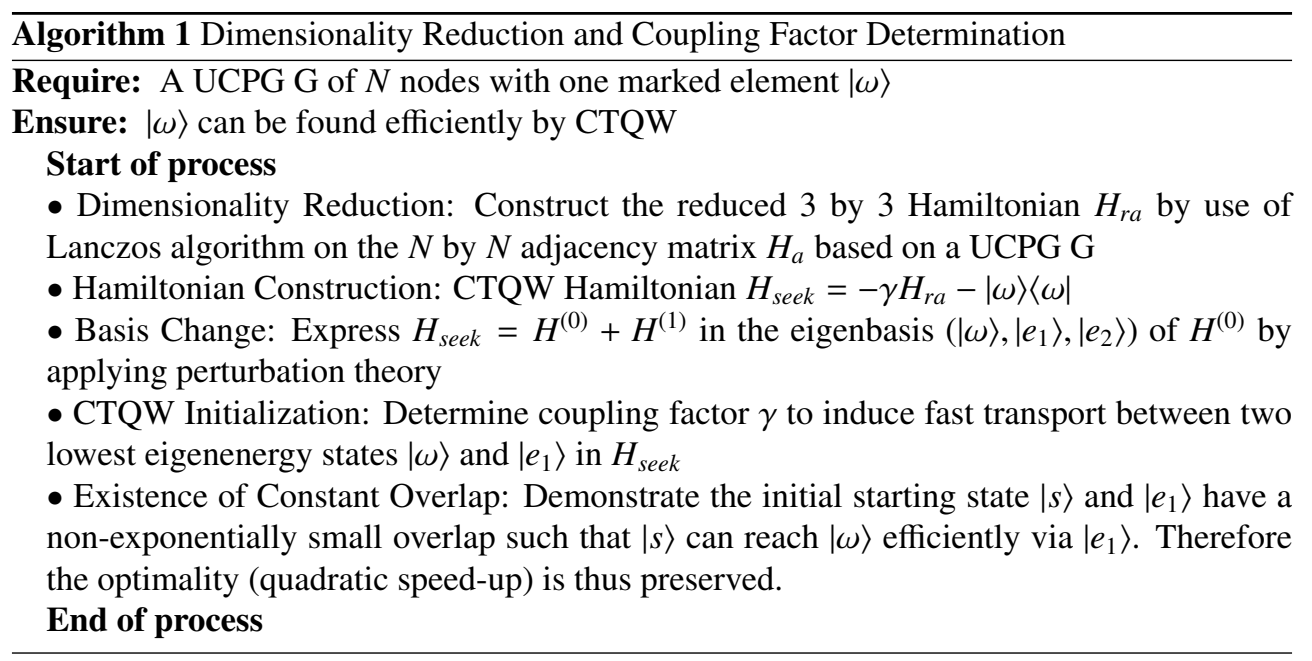

\subsection{Dimensionality Reduction}

Without loss of generality, let us assume the marked vertex $|\omega\rangle$ is in $V_{0}$ and $m_{0} \gg 1$. The original adjacency matrix is

$$
\begin{aligned}
H_{a} & =\sum_{i \in V, i \notin V_{0}}|i\rangle\left\langle\omega\left|+\sum_{j \in V_{0}, j \neq \omega} \sum_{i \in V, i \notin V_{0}}\right| i\right\rangle\langle j| \\
& +\sum_{k=1}^{P} \sum_{j \in V_{k}} \sum_{i \in V, i \notin V_{k}}|i\rangle\langle j| .
\end{aligned}
$$


With renormalization, the subspaces are spanned by $|\omega\rangle,\left|S_{V_{0}-\omega}\right\rangle,\left|S_{V_{1}}\right\rangle, \cdots,\left|S_{V_{P}}\right\rangle$ where

$$
\left|S_{V_{0}-\omega}\right\rangle=\frac{1}{\sqrt{m_{0}-1}} \sum_{i \in V_{0}, i \neq \omega}|i\rangle, \quad\left|S_{V_{i}}\right\rangle=\frac{1}{\sqrt{m_{i}}} \sum_{j \in V_{i}, i \neq 0}|j\rangle .
$$

Define $\left|S_{\bar{V}_{0}}\right\rangle=\frac{1}{\sqrt{N-m_{0}}} \sum_{i \in V, i \notin V_{0}}|i\rangle$, then by using Lanczos algorithm and the fact that non-solution partitions are of same size, the reduced adjacency Hamiltonian $H_{r a}$ in $\left(|\omega\rangle,\left|S_{V_{0}-\omega}\right\rangle,\left|S_{\bar{V}_{0}}\right\rangle\right)$ basis is

$$
H_{r a}=\left[\begin{array}{ccc}
0 & 0 & \sqrt{N-m_{0}} \\
0 & 0 & \sqrt{\left(N-m_{0}\right)\left(m_{0}-1\right)} \\
\sqrt{N-m_{0}} & \sqrt{\left(N-m_{0}\right)\left(m_{0}-1\right)} & N-m_{0}-m_{1}
\end{array}\right]
$$

The entry $H_{r a}(3,3)$ can be easily verified because of the uniform size of non-solution partitions and $(P-1) \times m_{1}=N-m_{0}-m_{1}$.

\subsection{CTQW Construction}

Let $\alpha=\frac{m_{0}}{N}$ and $\alpha_{1}=\frac{m_{1}}{N}$. Since $H_{r a}$ expressed in the $\left(|\omega\rangle,\left|S_{V_{0}-\omega}\right\rangle,\left|S_{\bar{V}_{0}}\right\rangle\right)$ basis captures the same dynamics as $H_{a}$, the Hamiltonian of a CTQW [5] is

$$
H_{\text {seek }}=-\gamma H_{r a}-|\omega\rangle\langle\omega|
$$

where $\gamma$ is the coupling parameter. Let

$$
H_{\text {seek }}=H^{(0)}+H^{(1)}
$$

by Eqn. $(3,4)$ we know ${ }^{1} 2$

$$
\begin{gathered}
H^{(0)}=\left[\begin{array}{ccc}
-1 & 0 & 0 \\
0 & 0 & -\gamma N \sqrt{\alpha(1-\alpha)} \\
0 & -\gamma N \sqrt{\alpha(1-\alpha)} & -\gamma N\left((1-\alpha)-\frac{P \alpha_{1}^{2}}{1-\alpha}\right)
\end{array}\right], \\
H^{(1)}=\left[\begin{array}{ccc}
0 & 0 & -\gamma \sqrt{(1-\alpha) N} \\
0 & 0 & 0 \\
-\gamma \sqrt{(1-\alpha) N} & 0 & 0
\end{array}\right] .
\end{gathered}
$$

It differs from the result in [4] because of the existence of a self-loop entry for the basis vector $S_{\bar{V}_{0}}$ in $H^{(0)}$. Because of this entry, it imposes a stronger constraint of equal size for nonsolution partitions for feasible systematic dimensionality reduction. In Theorem 1, we show how to express a reduced Hamiltonian $H_{\text {seek }}$ in the basis of its major matrix via perturbation theory.

Theorem 1 For simplicity, let us name $H_{\text {seek }}$ as $H$. Given a reduced Hamiltonian $H=H^{(0)}+$ $H^{(1)}$ in the $\left(|\omega\rangle,\left|b_{1}\right\rangle,\left|b_{2}\right\rangle\right)$ basis where

$$
H^{(0)}=\left[\begin{array}{ccc}
-1 & 0 & 0 \\
0 & 0 & v_{1} \\
0 & v_{1} & v_{3}
\end{array}\right], \quad H^{(1)}=\left[\begin{array}{ccc}
0 & 0 & v_{2} \\
0 & 0 & 0 \\
v_{2} & 0 & 0
\end{array}\right]
$$

${ }^{1}$ Clear $\alpha_{1}=(1-\alpha) / P=m_{1} / N$

${ }^{2}$ Entry $(3,3)$ at $H^{(0)}$ is thus $-\gamma N((1-\alpha)-(1-\alpha) / P)=-\gamma\left(N-m_{0}-m_{1}\right)$ 
$v_{1}$ and $v_{2}$ are negative numbers and $v_{3}$ is a non-positive number where $v_{1} / v_{2}=\sqrt{N \alpha} \geq 1$. Let the eigenvectors basis of $H^{(0)}$ be $\left(|\omega\rangle,\left|e_{1}\right\rangle,\left|e_{2}\right\rangle\right)$. Let $\kappa=\frac{v_{3}}{v_{1}}>0$ and $\beta_{ \pm}=\frac{\kappa \pm \sqrt{\kappa^{2}+4}}{2}$, then eigenvector $\left|e_{1}\right\rangle=\frac{\left(\left|b_{1}\right\rangle+\beta_{+}\left|b_{2}\right\rangle\right)}{\sqrt{1+\beta_{+}^{2}}}$ and eigenvector $\left|e_{2}\right\rangle=\frac{\left(\left|b_{1}\right\rangle+\beta_{-}\left|b_{2}\right\rangle\right)}{\sqrt{1+\beta_{-}^{2}}}$ with corresponding eigenvalues $\lambda_{ \pm}=v_{1} \beta_{ \pm}$. $H$ expressed in the $\left(|\omega\rangle,\left|e_{1}\right\rangle,\left|e_{2}\right\rangle\right)$ eigenbasis is

$$
H=\left[\begin{array}{ccc}
-1 & v_{2} \frac{\beta_{+}}{\sqrt{\beta_{+}^{2}+1}} & v_{2} \frac{\beta_{-}}{\sqrt{\beta_{-}^{2}+1}} \\
\frac{v_{2} \sqrt{\beta_{+}^{2}+1}}{\beta_{+}-\beta_{-}} & \lambda_{+} & 0 \\
\frac{-v_{2} \sqrt{\beta_{-}^{2}+1}}{\beta_{+}-\beta_{-}} & 0 & \lambda_{-}
\end{array}\right]
$$

Proof: Let $\left|e^{\prime}\right\rangle=\left|b_{1}\right\rangle+\beta\left|b_{2}\right\rangle$ be an eigenvector of $H^{(0)}$ with eigenvalue $\lambda$. After some calculation we obtain $\lambda=\beta v_{1}$ where where

$$
\beta=\frac{\kappa \pm \sqrt{\kappa^{2}+4}}{2}, \quad \kappa=\frac{v_{3}}{v_{1}} .
$$

Let eigenvector $\left|e_{1}^{\prime}\right\rangle=\left|b_{1}\right\rangle+\beta_{+}\left|b_{2}\right\rangle$ with eigenvalue $\lambda_{+}$and eigenvector $\left|e_{2}^{\prime}\right\rangle=\left|b_{1}\right\rangle+\beta_{-}\left|b_{2}\right\rangle$ with eigenvalue $\lambda_{-}$, by renormalization we have

$$
\left|e_{1}\right\rangle=\frac{\left|e_{1}^{\prime}\right\rangle}{\sqrt{\beta_{+}^{2}+1}}, \quad\left|e_{2}\right\rangle=\frac{\left|e_{2}^{\prime}\right\rangle}{\sqrt{\beta_{-}^{2}+1}}
$$

By basis change and Eqn.(8) with $H^{(1)}$ in the $\left(|\omega\rangle,\left|e_{1}\right\rangle,\left|e_{2}\right\rangle\right)$ eigenbasis, , we obtain $H^{(1)}|\omega\rangle$ as

$$
v_{2}\left|b_{2}\right\rangle=\frac{v_{2} \sqrt{\beta_{+}^{2}+1}}{\beta_{+}-\beta_{-}}\left|e_{1}\right\rangle+\frac{-v_{2} \sqrt{\beta_{-}^{2}+1}}{\beta_{+}-\beta_{-}}\left|e_{2}\right\rangle .
$$

Hence, the Hamiltonian H can be expressed as shown in Eqn. (9).

Lemma 1 Given a derived reduced Hamiltonian $H$ written in the $\left(|\omega\rangle,\left|e_{1}\right\rangle,\left|e_{2}\right\rangle\right)$ basis as shown in Theorem 1, we then know that (a) Hamiltonian $H$ is symmetric and (b) $\beta_{+}>0>\beta_{-}$ and $\lambda_{+}<0, \lambda_{-}>0$.

Proof: With the value of $\beta_{ \pm}$as shown in Theorem 1, we know $\beta_{+} \beta_{-}=-1$ and

$$
\begin{aligned}
& \beta_{+}\left(\beta_{+}-\beta_{-}\right)=\beta_{+}^{2}+1, \\
& \beta_{-}\left(\beta_{+}-\beta_{-}\right)=-\left(1+\beta_{-}^{2}\right) .
\end{aligned}
$$

With this observation, we can immediately conclude that

$$
v_{2} \frac{\beta_{+}}{\sqrt{\beta_{+}^{2}+1}}=\frac{v_{2} \sqrt{\beta_{+}^{2}+1}}{\beta_{+}-\beta_{-}}, \quad v_{2} \frac{\beta_{-}}{\sqrt{\beta_{-}^{2}+1}}=\frac{-v_{2} \sqrt{\beta_{-}^{2}+1}}{\beta_{+}-\beta_{-}} .
$$

Therefore, the property (a) that $H$ is symmetric is proved. For property (b), it is immediate since $\sqrt{\kappa^{2}+4}>\kappa>0$ and $v_{1}<0$.

For simplicity, let

$$
\delta_{1}=v_{2} \frac{\beta_{+}}{\sqrt{\beta_{+}^{2}+1}}, \quad \delta_{2}=v_{2} \frac{\beta_{-}}{\sqrt{\beta_{-}^{2}+1}} .
$$


By use of Lemma 1, H can be written in the $\left(|\omega\rangle,\left|e_{1}\right\rangle,\left|e_{2}\right\rangle\right)$ basis as

$$
H=\left[\begin{array}{ccc}
-1 & \delta_{1} & \delta_{2} \\
\delta_{1} & \lambda_{+} & 0 \\
\delta_{2} & 0 & \lambda_{-}
\end{array}\right]
$$

where $|\omega\rangle$ and $\left|e_{1}\right\rangle$ can form the basis for the two states of the lowest eigenvalue.

Theorem 2 Given a Hamiltonian $H$ in the form shown in Lemma 1, it is desirable to have $\lambda_{+}=-1$ such that $|\omega\rangle$ and $\left|e_{1}\right\rangle$ form the basis for the two states of the lowest eigenvalue. Since $v_{1}=-\gamma N(\sqrt{\alpha(1-\alpha)})$ then the degeneracy between site energies of $|\omega\rangle$ and $\left|e_{1}\right\rangle$ facilitates transport between these two low energy states, hence $\gamma=\left(N \sqrt{\alpha(1-\alpha)} \beta_{+}\right)^{-1}$. The transport between $|\omega\rangle$ and $\left|e_{2}\right\rangle$ is prohibited since $\delta_{2}$ is much smaller than $\lambda_{-}$.

Proof: Since we desire to have faster transport between the lowest eigen energy states, we need to set

$$
\lambda_{+}=v_{1} \beta_{+}=-1
$$

With the fact that $v_{1}=-\gamma N(\sqrt{\alpha(1-\alpha)})$, we need to set

$$
\gamma=\left(N \sqrt{\alpha(1-\alpha)} \beta_{+}\right)^{-1}
$$

From Eqn. $(16,17)$ and $\lambda_{-}$, we know $\delta_{2}$ is much smaller than $\lambda_{-}$because

$$
\frac{\delta_{2}}{\lambda_{-}}=\frac{v_{2}}{v_{1} \sqrt{\left(\beta_{-}^{2}+1\right)}}=\frac{1}{\sqrt{\alpha N\left(\beta_{-}^{2}+1\right)}}<\frac{1}{\sqrt{\alpha N}}{ }^{3} .
$$

For a given UCPG G, by use of Eqn.(6-8), we can properly bound $\kappa$ as

$$
\kappa=\frac{v_{3}}{v_{1}}=\frac{\sqrt{(1-\alpha)}\left(\frac{P-1}{P}\right)}{\sqrt{\alpha}}, \text { then } 0 \leq \kappa<\sqrt{\frac{1-\alpha}{\alpha}} .
$$

\subsection{From Constant Overlap to Optimality Preserving}

For a CTQW based on $H_{\text {seek }}$, we need to decide the value of coupling parameter $\gamma$ to ensure the optimal performance of the underlying quantum walk is preserved. If the coupling parameter $\gamma$ is wrongly chosen, the underlying CTQW search might not remain optimal, i.e. its quadratic speed-up might be lost. The determination process of correct $\gamma$ is shown in Theorem 2. Theorem 3 is an extension of Theorem 2 to various cases with respect to the values of variable $P$ and variable $\alpha$.

Theorem 3 Given a UCPG $G=\left(V_{0}, V_{1}, \cdots, V_{P}\right)$ and its adjacency matrix Hamiltonian $H_{a}$ in the $\left(|\omega\rangle,\left|b_{1}\right\rangle,\left|b_{2}\right\rangle\right)$ basis where $N=\sum_{i=0}^{P}\left|V_{i}\right|$, we can obtain the reduced search Hamiltonian $H_{\text {seek }}$ in a new eigenbasis $\left(|\omega\rangle,\left|e_{1}\right\rangle,\left|e_{2}\right\rangle\right)$ by use of Theorem 1 for constructing the underlying CTQW. We can then use Thereom 2 to determine the coupling factor $\gamma=\left(N \sqrt{\alpha(1-\alpha)} \beta_{+}\right)^{-1}$. The chosen $\gamma$ ensures the underlying CTQW remains optimal.

Proof: There are two aspects that we need to address to show that the optimality $O(\sqrt{N})$ is preserved. One (1) is fast search speed and low escaping speed while the other is (2) the overlap between $\left|e_{1}\right\rangle$ and the initial system state $|s\rangle$ (a uniform superposition) as it determines

\footnotetext{
${ }^{3}$ since $m_{0} \gg 1, \alpha=m_{0} / N$, then $\alpha N \gg 1$
} 
how many times we need to repeat the experiment.

The search speed is determined by the dynamics between fast transport non-solution $\left|e_{1}\right\rangle$ and solution state $|\omega\rangle$, i.e. $\left|e_{1}\right\rangle \rightarrow|\omega\rangle$. The degenerate eigenspace formed by $|\omega\rangle$ and $\left|e_{1}\right\rangle$ captures the dynamics between those two states. The escape speed is from solution $|\omega\rangle$ to undesirable non-solution states $\left|e_{2}\right\rangle$. From Eqn.(17), we know that $\delta_{1}$ is responsible for the search speed and $\delta_{2}$ is responsible for escape speed. In Eqn.(20)We have shown that $\delta_{2}$ is small with respect to $\lambda_{-}$, the escape speed is small. By use of Eqn.(16), we know that

$$
\left|\delta_{1}\right|=\left\langle e_{1}\left|H_{\text {seek }}\right| \omega\right\rangle=\left|\frac{v_{2} \beta_{+}}{\sqrt{\beta_{+}^{2}+1}}\right|=\left|\frac{-1}{\sqrt{\alpha N\left(\beta_{+}^{2}+1\right)}}\right|
$$

because

$$
v_{2}=-\gamma \sqrt{N(1-\alpha)}, \gamma=\left(N \sqrt{\alpha(1-\alpha)} \beta_{+}\right)^{-1} .
$$

Hence, we obtain the running time

$$
T_{\text {run }}=\frac{\pi}{2\left|\delta_{1}\right|}=\frac{\pi}{2} \sqrt{\alpha N\left(\beta_{+}^{2}+1\right)} .
$$

Let us verify that the running time $T_{\text {run }}$ remains optimal in different settings of UCPG $\mathrm{G}$ when the coupling factor $\gamma$ is chosen based on Theorem 2. Briefly speaking, with a fixed search space of size $N$, the configuration of a UCPG G is controlled by variable P and variable $\alpha$. We will discuss different settings based on those two variables.

Case 1: $P=1$

This is a typical complete bipartite graph as seen in [4]. We immediately know that $K=0$ since $\alpha_{1}=1-\alpha$ from Eqn.(21). This leads to $\beta_{+}=1$ from Eqn.(10). Because of that, no matter what value of $\alpha$ is, $T_{r u n}$ at Eqn.(24) holds its quadratic speed-up.

Case 2: $2 \leq P \leq N-1$ and $\alpha \propto \frac{1}{N}$

By Eqn.(21), we know that $\kappa \propto \sqrt{N-1}$ and by Eqn.(10), we know $\beta_{+} \propto \sqrt{N-1}$. By plugging in the values of $\alpha$ and $\beta_{+}, T_{\text {run }}$ at Eqn.(24) still holds its quadratic speed-up.

Case 3: $2 \leq P \leq N-1$ and $\alpha \propto 1$ (such as $\frac{N-1}{N}$ )

By Eqn.(21), we know that $k \propto 1 / \sqrt{N-1}$; by Eqn.(10), we know

$$
\beta_{+} \propto \frac{(1 / \sqrt{N-1})+\sqrt{(1 / N-1)+4}}{2} \simeq 1
$$

when $N$ is large. By plugging in the values of $\alpha$ and $\beta_{+}, T_{\text {run }}$ at Eqn.(24) still holds its quadratic speed-up.

Case 4: $2 \leq P \leq N-1$ and $\alpha$ is some constant (non-extreme values):

Immediately we know $\kappa$ and $\beta_{+}$are some constants that would not affect the complexity. Hence, $T_{r u n}$ at Eqn.(24) still holds its quadratic speed-up.

However, the $T_{\text {run }}$ above assumes that we start the search from eigenstate $\left|e_{1}\right\rangle$ to find $|\omega\rangle$, i.e. $\left|e_{1}\right\rangle \rightarrow|\omega\rangle$, which is not the case because we start from $|s\rangle$. Hence, at $T_{r u n}$ the success 
probability of observing $|\omega\rangle$ is the overlap between $\left|e_{1}\right\rangle$ and $|s\rangle$. The success probability is ${ }^{4}$

$$
P_{O}=\left|\left\langle e_{1} \mid s\right\rangle\right|^{2}=\left|\frac{\sqrt{\frac{\alpha}{\beta_{+}^{2}}-\frac{1}{\beta_{+}^{2} N}}+\sqrt{1-\alpha}}{\sqrt{1+\frac{1}{\beta_{+}^{2}}}}\right|^{2} .
$$

Therefore $1 / P_{O}$ is the number of times we need to repeat the experiment. We need to show that $P_{O}$ is some constant such that it would not affect the total complexity under the Big $O$ notation. By examining the four cases listed in Theorem 3 and the corresponding values of $\alpha$ and $\beta_{+}$into Eqn.(26), we know that $P_{O}$ remains as some constant that is not exponentially small. The total runtime is $T_{r u n} \times \frac{1}{P_{O}}$ where $T_{r u n}$ holds quadratic speed-up and $\frac{1}{P_{O}}$ is some constant that is not large (not scaling with $N$ ), the complexity still holds the quadratic speed-up. Therefore, we know that the chosen $\gamma=\left(N \sqrt{\alpha(1-\alpha)} \beta_{+}\right)^{-1}$ ensures that the underlying CTQW remains optimal.

\section{Noise Error Patterns and Optimality Preserving}

Now we consider how to keep the dimensionality reduced quantum walk search optimal by characterizing the noise patterns in the system. The noises can be introduced due to the precision limitation and the noisy environment. For instance, not all numbers have a perfect binary representation and the approximated numbers would cause perturbation. Let matrix $H_{a}^{\prime}$ be the closest Hamiltonian to $H_{a}$ that can be prepared by an available quantum system of limited precision. In the remaining of this section, we examine the effect of (1) systematic errors, (2) static errors, and (3) non-static errors on CQTW and the coupling factor while the goal is to keep the search by CTQW optimal and to keep the feasibility of systematic dimensionality reduction. For simplicity, let us assume we are working on only complete graphs (CG) for the noise characterization.

\subsection{Systematic Disorder}

If the error is systematic, that is each adjacency matrix entry that connects two different sites in $H_{a}^{\prime}$ suffers an $\epsilon$ error in comparison to the original $H_{a}$. It is clear to see $H_{a}^{\prime}=(1-\epsilon) H_{a}$. This $N$ by $N H_{a}^{\prime}$ matrix can be efficiently reduced to $(1-\epsilon) H_{r a}$ smoothly by use of Lanczos algorithm. This can applies to all UCPG graphs, including CG, CBG and SG. The new coupling factor $\gamma^{\prime}$ scales by a factor of $\frac{\gamma}{1-\epsilon}$ accordingly to keep the search optimal with

$$
\gamma^{\prime}=\left(N\left(\sqrt{\alpha(1-\alpha)} \beta_{+}\right)(1-\epsilon)\right)^{-1}
$$

In reality, the distribution of errors is seldom perfectly systematic when environmental noise is considered. When an arbitrary noise distribution is introduced into a quantum system of a higher dimension, the task becomes daunting. The computation complexity exponentiates due to high dimensionality and the irregularity in noise distribution.

${ }^{4}$ Simply compute their inner product and we know that $|S\rangle=\frac{|\omega\rangle+\sqrt{m_{0}-1}\left|S_{V_{0}-\omega}\right\rangle+\sqrt{N-m_{0}}\left|S_{\bar{V}_{0}}\right\rangle}{\sqrt{N}}$ 


\subsection{Static Diagonal Disorder}

Environmental assisted quantum search [6] suggests that naturally occurring open quantum system dynamics can be advantageous for a quantum algorithm based on quantum walks affected by static diagonal errors. The scenario considered is diagonal disorder due to imperfect Oracle. An imperfect Oracle that marks each node of the graph erroneously that non-solution node $j$ is marked with an energy $\epsilon_{j}$ while the solution node $\omega$ is marked with an energy $-1+\epsilon_{\omega}$. With the state $|s\rangle=\frac{1}{\sqrt{N}} \sum_{i=0}^{N-1}|i\rangle$, the perturbed unreduced search Hamiltonian with tuning factor $\gamma=1 / N$ is

$$
H_{\text {seek }}=-|\omega\rangle\langle\omega|-| s\rangle\left\langle s\left|+\sum_{i=0}^{N-1} \epsilon_{i}\right| i\right\rangle\langle i|
$$

where the static disorder $\epsilon_{i}$ are i.i.d random variables with mean 0 and standard deviation $\sigma<<1$. With degenerate perturbation theory, the reduced search Hamiltonian in the $\left\{|\omega\rangle,\left|s_{\bar{\omega}}\right\rangle\right\}$ basis is

$$
H_{\text {seek }}=\left[\begin{array}{cc}
-1+\epsilon_{\omega} & -1 / \sqrt{N} \\
-1 / \sqrt{N} & -1
\end{array}\right]
$$

where $\left|s_{\bar{\omega}}\right\rangle$ is the equal superposition of all nodes other than th solution node $|\omega\rangle$. The gap between the ground state and the first excited state of the perturbed Hamiltonian is

$$
\Delta=\sqrt{\epsilon_{\omega}^{2}+4 / N}
$$

With $\gamma=1 / N$, the success probability of the algorithm is

$$
P_{\omega}(t) \simeq \frac{\sin ^{2}(\Delta t)}{1+N \epsilon_{\omega}^{2} / 4}
$$

The maximum success probability is obtained when $t=\pi / \Delta \simeq O(\sqrt{N})$. From experimental aspect, this algorithm can always choose a fixed tuning factor, then the optimality is preserved by calculating the running time solely based on $\epsilon_{\omega}$ that is some variable with value between 0 and 1 . Interested readers can refer to this article [6] for technical details.

\subsection{Reducible Non-Diagonal Noise}

Suppose non-negative errors $\epsilon_{i j}$, where $\forall i, j \in[1, N]$ and $i \neq j$, occur across the original adjacency matrix $H_{a}$. To make this scenario not be mixed with the static diagonal disorder, $e_{i i}=0$, let the perturbed adjacency matrix be $H_{a}^{\prime}$ with $H_{a_{i j}}^{\prime}=H_{a_{i j}}-\epsilon_{i j}$ and let the index for state $|\omega\rangle$ be 1 in the system. Without loss of generality, let $|\omega\rangle$ be the solution state and the first normal basis vector.

In the first iteration of Lanczos, we know $\left\langle\omega \mid \omega_{1}\right\rangle=0$, where $\left|\omega_{1}\right\rangle=H_{a}^{\prime}|\omega\rangle=\sum_{i=2}^{N}(1-$ $\left.\epsilon_{i 1}\right)|i\rangle$. Let $\left|v_{2}\right\rangle=\frac{\left|\omega_{1}\right\rangle}{\left.\| \omega_{1}\right\rangle \|}$ be the second normal basis vector. In the second iteration, let $\left|\omega_{2}^{\prime}\right\rangle=$ $H_{a}^{\prime}\left|v_{2}\right\rangle$. For the Lanczos to terminate at this stage, the first condition is that $\left|\omega_{2}^{\prime}\right\rangle$ must be a linear combination of $|\omega\rangle$ and $\left|v_{2}\right\rangle$ that

$$
\left|\omega_{2}^{\prime}\right\rangle=c_{1}|\omega\rangle+c_{2}\left|v_{2}\right\rangle
$$

When the first condition is met, we have $\left\langle\omega_{2}^{\prime} \mid v_{2}\right\rangle=c_{2}$ since $\left|v_{2}\right\rangle \perp|\omega\rangle$. By running Lanczos algorithm, it is necessary to have the following second condition

$$
\left|\omega_{2}\right\rangle=\left|\omega_{2}^{\prime}\right\rangle-\left\langle\omega_{2}^{\prime} \mid v_{2}\right\rangle\left|v_{2}\right\rangle-\|\left|\omega_{1}\right\rangle \||\omega\rangle=0
$$


such that Lanczos algorithm terminates. It is thus desired to have $c_{1}=\|\left|\omega_{1}\right\rangle \|$ due to Eqn. $(32,33)$. The corresponding reduced Hamiltonian $H_{r a^{\prime}}$ in $\left(|\omega\rangle,\left|v_{2}\right\rangle\right)$ basis with noise is

$$
H_{r a^{\prime}}=\left[\begin{array}{cc}
0 & c_{1} \\
c_{1} & c_{2}
\end{array}\right]
$$

This immediately implies

$$
c_{1}=\sqrt{\sum_{i=2}^{N}\left(1-\epsilon_{i 1}\right)^{2}}
$$

for the second condition to be satisfied. Next we need to examine the constraint on $c_{1}, c_{2}$ such that the first condition is satisfied. We need to show that

$$
\left(H_{a}^{\prime}\left|v_{2}\right\rangle\right)=\frac{1}{\|\left|\omega_{1}\right\rangle \|}\left(\left(\sum_{i=2}^{N}\left(1-\epsilon_{i 1}\right)\left(1-\epsilon_{1 i}\right)|\omega\rangle\right)+\sum_{k=2}^{N} \sum_{i=2, i \neq k}^{N}\left(1-\epsilon_{k i}\right)\left(1-\epsilon_{i 1}\right)|k\rangle\right)
$$

is the same as Eqn. (32). This immediately gives us the requirement for $c_{2}$ the noise pattern that $\forall k \in[2, N]$

$$
\sum_{i=2, i \neq k}^{N}\left(1-\epsilon_{k i}\right)\left(1-\epsilon_{i 1}\right)=c_{2}\left(1-\epsilon_{k 1}\right)
$$

For $c_{1}$, by use of Eqn. $(35,36)$ and the fact that $c_{1}=\|\left|\omega_{1}\right\rangle \|$, we know $c_{1}^{2}$ has

$$
c_{1}^{2}=\sum_{i=2}^{N}\left(1-\epsilon_{i 1}\right)^{2}=\sum_{i=2}^{N}\left(1-\epsilon_{i 1}\right)\left(1-\epsilon_{1 i}\right) .
$$

\section{Discussion}

A high dimension Hamiltonian will not be feasbile to implement using near-term quantum technology if we try to encode the given original configuration. With our reduction scheme and coupling factor determination approach, we can implement the dynamics of the high dimension Hamiltonian with very few quantum bits and the quantum walker will remain optimal while searching on this reduced system. Furthermore, since quantum system is susceptible to the noise, to keep reduction scheme possible we characterize the noise pattern for three types of error distribution: systematic disorder, static diagonal disorder, and reducible non-diagonal disorder. For the first two cases, it is apparent that no specific pattern is required while for the reducible non-diagonal errors, we know the pattern must satisfy Eqn.(37,38) such that the pattern guarantees systematic dimensionality reduction.

\section{References}

[1] Y. Aharonov, L. Davidovich, N. Zagury, Physical Review A 48, 1687 (1993)

[2] E. Farhi, S. Gutmann, Physical Review A 58, 915 (1998)

[3] A.M. Childs, Communications in Mathematical Physics 294, 581 (2010)

[4] L. Novo, S. Chakraborty, M. Mohseni, H. Neven, Y. Omar, Scientific reports 5 (2015)

[5] A.M. Childs, J. Goldstone, Physical Review A 70, 022314 (2004)

[6] L. Novo, S. Chakraborty, M. Mohseni, Y. Omar, arXiv preprint arXiv:1710.02111 (2017) 